\title{
CONGENITAL HYDRONEPHROSIS
}

IN A BOY FOUR YEARS OLD;

REPEATEDLY TAPPED. RECOVERY.

BY

THOMAS HILLIER, M.D. LOND.,

phrsiciay to the hospital for sick childrea, physician to the

SKIN DEPARTMENT OF UNIVERSITY COLLEGE HOSPITAL, AND

MEDICAL OFFICER OF HEALTH FOR ST. PANCRAS.

Received Dec. 23rd, 1861.-Read March 14th, 1865.

J. R- first came under my notice in March, 1863. He was then three years and four months old.

His mother stated that his stomach had been large ever since birth; when three months old a doctor who saw him recommended tapping, but the parents would not allow it.

When sixteen months old he was a patient in the Leeds Infirmary, and the doctor there thought it should be tapped. The operation was decided on, but in three days the swelling had subsided so much that the operation was not performed.

For twelve months he had been much in the condition in which I first saw him, the abdomen, however, being sometimes larger than at others; at different times the circumference had varied from twenty-four to twenty-seven inches. The child had always had a large appetite; nothing abnormal had been observed in the alvine or urinary secre- 
tions. His father's family was consumptive, and he had several brothers and sisters, all of whom were said to be delicate.

On April 2nd the following note was made of his condition.

Patient is small for his age; he has a rickety head and limbs. He sits up in bed, seems comfortable, and has no dyspnœa. His limbs are moderately nourished. Pulse 104, tolerably full and regular; respirations thirty in the minute.

The heart's apex is raised, beating almost close to the nipple; its sounds are normal; lateral diameter of thorax narrow from rickets.

Abdomen very large and tense; skin smooth and shining; superficial veins extremely large and distinct. There is lateral bulging, more marked on the right than on the left side. The ribs are pushed up, and the ensiform cartilage is tilted upwards; the greatest circumference of the abdomen is at a point midway between the ensiform cartilage and the umbilicus, where it measures twenty-six inches. From the tip of the ensiform cartilage to the umbilicus is seven inches and a half. The swollen abdomen fluctuates very distinctly on palpation. Between the ensiform cartilage and the umbilicus there is a tympanitic percussion-note; below the umbilicus the percussion-note is duller, and in the right flank there is absolute dulness.

Over the abdomen generally there is an irregular doughy sensation on manipulation, but in the right hypochondrium there is a sense of firm resistance. The liver and spleen cannot be detected, and no tumour can be made out. There is no sign of dropsy except in the abdomen; urine is acid, free from albumen, having a specific gravity of 1016, and is passed in fair amount.

The case at this time was not understood, and it was supposed there was fluid in the peritoneal sac, but from what cause could not be conjectured. There was no reason to suspect hepatic or mesenteric disease.

A more careful consideration of the physical signs would have shown that the fluid accumulation was not in the 
peritoneal cavity. The extreme dulness of the percussionnote, the firm resistance offered on pressure in the right flank, and the want of symmetry in the swelling, were not compatible with simple ascites.

Under the false impression just referred to the patient was treated with diuretics and purgatives. But no benefit resulted. The abdomen increased in size until it measured twenty-eight inches and a half in circumference, and it was noted that the right half of the abdomen measured an inch and a half more than the left. This was on the 16th of April. His abdomen was so heavy that when he was lying down he could not raise himself up again.

On the 17th of May the abdomen had diminished in size, and measured about twenty-four inches. As his health was not suffering, and his respiration not much interfered with, he was allowed to go home.

On the 8th February, 1864, he again came under my care at the Hospital for Sick Children, and on the 17th the following note was made of his condition.

His general health is tolerably good, though his limbs are weak, and from the weight of his abdomen he is unable to walk.

The abdomen is almost globular, but the swelling is greater below than above the navel, and greater on the right side than on the left. The circumference midway between the navel and tip of the xiphoid cartilage is twenty-seven inches. The umbilicus is not protruded, an enlarged vein runs from the right side of epigastrium towards the navel. Midway between the navel and the end of the ensiform cartilage there is a distinct sulcus (more marked on the left than on the right side) moving with respiration. This sulcus is found on close examination to correspond to the lower portion of the transverse colon, which is movable, and can be pushed down to within an inch and a half of the navel; it lies on a firm globular swelling, which is elastic and fluctuating. Something like the cæcum can be felt in the right iliac fossa in front of the same mass. The surface of the swelling is everywhere smooth and even, and the percussion-note is absolutely dull. There 
is perfect fluctuation throughout every part of it. The prominence of the tumour can be most distinctly seen and felt in the right lumbar region; there is nothing corresponding to it on the left side. Distinct fluctuation is transmitted from the navel to the right lumbar region. From the notch of the sternum to the end of the xiphoid cartilage measures three inches and five-eighths; from the xiphoid to the navel seven inches and three-eighths, and from the navel to the pubes measures three inches and five-eighths. The upper limit of hepatic dulness is one finger's breadth below right nipple. The heart's impulse is felt just above and inside the left nipple. The urine is clear, pale, and free from albumen.

It was now obvious that the boy had a large cyst, with not very thick walls, springing from the right side of his abdomen, very likely connected with the right kidney, and that the large intestines were in front of it.

It was resolved to tap the cyst to relieve the patient of his burden, which prevented his walking and interfered with his respiration.

A trocar was introduced half an inch below and an inch and a half to the right of the navel. There escaped 102 fluid ounces of clear yellowish fluid, faintly acid in reaction, having a specific gravity 1008 . It contained no albumen and there was no sediment. It had all the characters of dilute urine.

On chemical analysis it was found to contain 20.304 grammes or nearly 313 grains of urea ; 5.9 grammes or 81 grains of chloride of sodium, and 0.457 gramme or 7 grains of uric acid. It also contained phosphates and sulphates.

From this analysis it was concluded that the cyst was the dilated pelvis of the right kidney, due probably to malformation or obliteration of the ureter on that side.

After the operation he was left undisturbed, and there were no bad symptoms consequent upon the tapping. For twenty-four hours after it he had retention of urine, and the bladder was relieved by the catheter. 
Four days after the operation, the abdomen measured twenty-tbree inches and a half; his tongue was clean and moist, and his skin cool; pulse 118.

On 25th of February, a week after the operation, the abdomen was much flatter than it had been; it measured from the xiphoid cartilage to the navel only five inches and a half instead of seven and three-eighths, and in its greatest circumference twenty-three inches and seven-eighths.

On palpation of the abdomen, the condition of the parts was much less obvious than before. Still, a line was made out passing from right to left, and from above downwards, separating a less resistant upper part from a more resistant lower one; the latter was dull on percussion, while the former was tympanitic; owing to the flaccid state of the abdominal walls, fluctuation could not be well made out. Fæcal masses could be felt in the ascending, transverse, and descending colon. The heart's apex was felt a little nearer the middle line, but not lower than before.

The swelling soon increased, and by the 5th of March the abdomen measured twenty-five inches and three-quarters.

On the 15th of March an experiment was instituted to determine whether the fluid which was re-collecting in the cyst was of the same character as the urine. As the abdomen now measured twenty-nine inches, it was thought desirable again to tap him; but before doing so he was to take for two days ten grains of the ferrocyanide of potassium twice a day.

On the 16th the urine gave a very dark colour on the addition of the sesquichloride of iron, owing to the ferrocyanide passing off by the kidney.

On the 18th a small quantity of fluid was let out of the cyst, in order to compare it with what was drawn off by the first operation, and to see if it contained ferrocyanide of potassium.

It was now found to be neutral in reaction, slightly turbid from pus, and it contained a trace of albumen. It exhibited no reaction with the sesquisalts of iron. It contained proportionately only half as much urea as the first specimen. 
The circumstance that the fluid drawn off contained no ferrocyanide, although the urine did, and that the urea had diminished in amount, made it doubtful whether the fluid which was re-formed had been secreted by the kidney or was from the wall of the cyst alone; on the latter supposition the urea which it contained must have come from the fluid left after the first operation.

By the 29th of March the abdomen had attained a size as large as ever it had; it measured twenty-nine inches and three-quarters. After a consultation it was thought advisable again to tap the cyst, and establish a permanent fistula. At this time the boy had frequent attacks of dyspnoea at night, which obliged him to sit up in bed.

On the 7th of April a small piece of caustic potash was applied to the abdominal wall in the situation of the former punctures, with the view of setting up adhesions between the cyst and the parietes, before opening the cyst.

During the two following days the boy became so weak and ill that it was not considered safe to wait for the gradual action of the caustic; paracentesis was accordingly performed with a trocar, and after drawing off the contents, the canula was left in the sac, and its free extremity was corked up. About seventy-four ounces of fluid were drawn off in this operation. It was now of a deep chocolate colour, nearly opaque, alkaline, and depositing about one quarter of albumen on boiling and the addition of nitric acid.

Under the microscope numerous blood-discs and pyoid cells were seen. It contained relatively more urea than the first specimen, namely, 6.5 grammes in 1000 cubic centimeters of fluid instead of 6.2 grammes in the same quantity.

For five days-from the 13th to the 17th March-the urine was carefully saved, and found to exhibit the following characters :-it was free from albumen, sometimes faintly acid and sometimes neutral in reaction. The average quantity passed in twenty-four hours during the five days was only 306 cubic centimeters, or between ten and eleven ounces. Its specific gravity varied from 1019 to 1026 . He passed on the 
average $7 \cdot 8$ grammes, or 120 grains of urea per diem, and of chloride of sodium 1.524 gramme, or 23.4 grains.

Considering his weight (twenty-nine pounds) and age, there was a decided deficiency of water and solids in the urine; there was not more than half the normal amount of water, about two thirds of the normal quantity of urea, and one quarter of the normal amount of chlorine; estimated on the scale given by Dr. Parkes in his treatise on the urine.

The fluid drawn off by tapping contained urea in the proportion of rather less than a third of what his urine passed by the bladder contained.

On the 10th April (the day after the third tapping) he was very ill. His pulse was 190 ; he complained of much headache and abdominal pain. His temperature was $104.75^{\circ}$ Fahr.; tongue furred, white, and dry.

The canula was withdrawn, and a piece of gum elastic catheter substituted for it. Fifty-two ounces of fluid were drawn off, of a specific gravity of 1008, and with other characters exactly similar to what had been obtained on the previous day. Almost directly after this he passed by the urethra about eight ounces of fluid, specific gravity 1015, of a colour midway between that of the fluid drawn from the cyst and that of his urine. In half an hour later he passed five ounces of fluid almost identical in appearance with that of the cyst. It had a specific gravity 1012 ; contained an albuminous precipitate, when boiled and acidulated, filling nearly one quarter of the test-tube, and it had an alkaline reaction.

The previous day his urine had been clear, faintly acid, without albumen, and had a specific gravity 1017.

This was the first occasion on which we had any distinct evidence that the cyst and the bladder ever communicated, although the mother's statement that, on several occasions after extreme distension of the abdomen, the swelling had suddenly subsided, made it probable that such a communication had existed.

On the 11th April there was a marked increase in the quantity of urine passed, and it was of a pale-brown colour 
and faintly turbid; had a specific gravity 1014, and contained a trace of albumen. It was quite clear from these marked changes in the urine that a free communication had been suddenly opened up between the bladder and the cyst.

On the 13th April he was better, had no pain; pulse 156; temperature $99^{\circ}$. A good deal of fluid dribbled from the wound. Urine very slightly turbid, was faintly acid, and contained a mere trace of albumen.

On 15th April, pulse 130; much emaciated. There was much fluid, sometimes thin sometimes thick, dribbling from the canula; the quantity was estimated at two quarts in twenty-four hours. The abdomen was not tender, and measured twenty-one inches and seven-eighths. He did not pass any urine for thirty-six hours. The cyst was now injected with warm water and a weak solution of Condy's fluid, to correct fotor and cleanse it.

On 21st April the boy's general condition was much the same. All the fluid that escaped from the cyst in twentyfour hours was saved, and measured 345 cubic centimeters or about 12 ounces; it was light-coloured, turbid, very fœtid, highly albuminous, and contained about thirty-six grains of urea. A large quantity of pus was also present in it.

On the next day the discharge from the cyst entirely ceased, and on introducing a probe to the wound it was obstructed at a distance of about an inch and three-quarters from the entrance. From this time the swelling again rapidly increased in size until, on the 2nd May, the abdomen measured twenty-seven inches and one-eighth. A tumour was now noticed in the left flank, just outside the quadratus lumborum, reaching from the twelth rib to the crest of the ilium, about two inches across. It was dull on percussion, did not fluctuate; the surface and edges felt even. This was no doubt the left kidney.

On the 7th May, the tension being very great, the abdominal 'wall gave way at the seat of the old punctures, and a quantity of fluid escaped. A long curved canula was now placed in the cyst with the view of keeping it permanently open. It was found impossible to do this; the 
fluid soon ceased to flow through the canula, and the wound healed.

On the 30th May it was determined to establish an opening by means of caustic potash in the right loin.

On the 6th June the abdomen measured twenty-nine inches and a half; the caustic had formed a deep slough, but had not opened into the cyst. A trocar was introduced through the eschar, and about two quarts of thin, fetid, purulent fluid escaped.

It was found impossible to keep this wound open; it healed up, and the boy's condition became much the same as before.

On 27th June he was regaining flesh and strength.

A little later he had an attack of measles, which he passed through without a drawback.

On 24th September it was noted that he had much improved in his general health; was quite fat, and could walk with slight support.

The abdomen measured twenty-five inches and three quarters in circumference, and from the ensiform cartilage to the navel five inches and a quarter. It was much softer and more yielding than it had been; it swayed over to the flanks to a certain extent, and was very flat on the top. The swelling in the left loin was not now to be felt distinctly.

The urine was saved for twenty-four hours, and found to have a specific gravity 1013, and to measure about seventeen fluid ounces. It was pale, turbid, and very fetid. The microscope showed-(1) prisms of triple phosphates; (2) vibriones; (3) pus and pyoid cells, and small casts containing pyoid cells, very fatty. He was now sent to the convalescent establishment at Mitcham.

Since that time he has continued to improve; can walk about well, and has gained flesh. The abdomen varies in size from time to time, but has never been so much distended as it was some months before.

On the 15th December he was looking quite fat and comfortable. The abdomen was flaccid, flattened in front, and prominent at the sides, especially on the right. A resistant vor. xIvIII. 
mass, dull on percussion, was felt on firm pressure before and behind, in the right lumbar region. The largest circumference of the abdomen was twenty-four inches.

His urine was of a light lemon colour, with a copious deposit of pus and triple phosphates, and was very offensive. After filtration, boiling, and the addition of nitric acid, there was no deposit, notwithstanding the presence of a large quantity of pus. This circumstance, namely, the absence of any indication of albumen by heat and nitric acid, I have also observed in urine which contained numerous blood-discs. It must be explained by supposing that the albumen is prevented from precipitation by some peculiar ingredient; or can it be that pus- and blood-cells may be found without any albumen, except what the cells themselves contain?

For the examination of the urine and many of the clinical observations in this case I am indebted to Mr. S. Gee, M.B., Assistant-Physician and Registrar to the Hospital for Sick Children.

Several cases are on record in which the ureter has been obstructed from birth, and has led to a dilated condition of the pelvis of the kidney. Most of these cases have died young, and the kidney bas not attained any large size.

A remarkable case is recorded by Rayer ("Traité des Maladies des Reins,' vol. iii), in which on the right side of the abdomen there was found an enormous cyst, having the anatomical relations of a kidney. This patient lived to the age of twenty-three years. The contents of the cyst were not analysed, so that we do not know whether it contained urinary ingredients.

Another curious case is reported by Rayer, of a youth who lived to the age of seventeen years, and was found after death to present double hydronephrosis; that on the left side being due to a valvular obstruction of the ureter, not completely cutting off communication between the kidney and bladder, and that on the right side being due to a stricture of the ureter.

In the case which I have reported there is obviously a cyst in which the renal secrétion accumulates; this cyst does not 
usually communicate freely with the bladder, but from time to time, when much distended, its contents are partially poured into that viscus; there is some renal glandular tissue existing on that side, as proved by the constant presence of large quantities of urea in the fluid; the cyst is probably sacculated, and there is not a free communication between all the parts of the cyst, as proved by the difficulty of completely emptying the cyst and of keeping open any fistula which is made. It would seem as if the loculus into which the canula passes is after a time shut off from the rest of the cyst.

Why was no ferrocyanide detected in the fluid of the cyst when it was present in the urine?

Thinking it possible that the presence of albumen in the cystic fluid might prevent the reaction of the ferrocyanide, I gave this salt to another patient who had albuminuria, and failed to detect it in his urine after he had taken twenty grains of the ferrocyanide of potassium.

This induced me to try the same experiment with gallic acid. I found that gallic acid could be detected in his urine after it had become albuminous, but no trace of it was found in the cystic fluid.

This shows either that transudation of gallic acid did not take place through the kidney of the right side, though it did on the left, or else that there was not a free communication between the part of the cyst in which the canula was inserted and that part into which the kidney first emptied itself.

The history of the case clearly proves that the ureter on the right side was often obstructed, but at other times allowed fluid to pass through it.

A valvular obstruction such as existed in one of Rayer's cases or in Dr. Broadbent's case, presently to be men. tioned, may be present, and it is possible that the valve may allow fluid to pass under certain conditions, and not under others.

A calculus might possibly be so placed as to cause an intermitting obstruction to the flow of urine down the ureter. I have not met with the record of any case in which a 
calculus was so situated as to dilate the kidney to such an extent as in this patient. The absence of pain is entirely opposed to this supposition.

The treatment adopted in this case has been of no service, although it would seem to be the course which the symptoms indicated as suitable. The distension was so great as seriously to endanger the boy's life, as well as to make his life miserable. When the diagnosis was once correctly made, it was obvious that tapping was the only remedy offering any chance of getting rid of the fluid; and inasmuch as the cyst rapidly refilled, the establishment of a permanent fistula appeared desirable, to prevent the necessity for repeated operations. This, however, was found to be impracticable, from the fluid after a short time ceasing to flow. The question was then raised whether the injection of the cyst with iodine was allowable. It was decided that this would be imprudent, seeing that there appeared to be a secreting kidney still existing, that the cavity was so large, and that there would be great risk of inflaming the peritoneum. The next point to consider was whether removal of the cyst as a whole might be attempted. It was thought that the cyst might be removed from behind. Would the risk be greater than that attending the operation of ovariotomy? The left kidney would, no doubt, be able to do the work of two kidneys. There is reason to believe that it is already hypertrophied, and does more than its share of duty. There would, however, I believe, be too great risk attending the operation for extirpation of the cyst, from interference with the sympathetic nerve near the semilunar ganglia, as well as from hæmorrhage and peritonitis. The removal of a kidney from the lower animals has, I believe, always proved fatal.

At present it seems probable that the patient may live for some years in comparative comfort, although much incommoded by the size of his abdomen, and liable at any time to greater accumulation of fluid. Paracentesis may from time to time be necessary, but should only be attempted in cases of extreme distension, seeing that nature usually provides a remedy when she is left to herself. The left kidney is very 
likely to suffer from sympathy and from the extra duty thrown upon it.

The irritation set up in the cyst by tapping, and the exhaustion accompanying it, would soon have put an end to his life had the operations been much oftener repeated. It is a very remarkable feature in this case that after such long continued pressure on the right kidney from accumulating fluid there should be left any renal structure capable of secreting urea.

The arteries of this little patient have undergone a peculiar degeneration, such as is usually seen only in old people. Most of the superficial arteries are very tortuous and visible, apparently from a loss of elasticity.

In new-born children hydronephrosis of one kidney has been several times observed.

Cases are reported by Bonet, in the 'Sepulcretum,' vol. ii, p. 290 ; by Billard, in his 'Traité des Maladies des Nouveaunés,' p. 434; and by Dr. Harley, in the ' Pathological Society's Transactions,' vol. xv, p. 146.

Double hydronephrosis in new-born children is very rare. A case was exhibited at the Académie de Médecine, in 1828, by M. Moreau; and a case due to obliteration of the urethra is reported by Billard, 'Traité des Maladies des Nouveaunés,' page 436.

A specimen was recently exhibited to the Pathological Society by Dr. Broadhent, and reported on by Dr. Harley, in which the ureters were "irregularly distended, so that when filled with water they looked almost sacculated. Water injected from below flowed into the pelvis of each kidneyinto the left with facility, into the right with difficulty. The pelvis of each kidney was much dilated, the right twice as much as the left; the former might hold seven or eight, the latter four, ounces of fluid. The parenchyma of the kidney was altered in form and condensed. No calculi or deposit of any kind was detected." The greater distension of the right kidney was found to be due to a circular fold of mucous membrane near the renal end of the right ureter, which acted as a valve and completely blocked up the passage until the 
ureter was fully dilated. Several imperfect valvular folds existed in both ureters. The bladder was dilated, but the urethra was not examined. This specimen was taken from a child three months old. The dilatation of the kidneys was, no doubt, congenital.

Cases of pyelitic distension in adults are not very uncommon. Accumulation takes place from temporary obstruction of the ureter, which is repeated at shorter or longer intervals. The following case came under my notice in November, 1855 :

A married woman, æt. 47 , had suffered for three or four years with pain in the right side of the abdomen, returning at irregular intervals. She had noticed, when the pain was most severe, an oval swelling in the right lumbar region, which disappeared as the pain subsided. She also stated that during the height of the attack her urine was very scanty, but afterwards it was very abundant and very turbid for some days.

When I first saw her, she was in great pain, her pulse was weak, and her extremities were cold. There was much tenderness in the loins, especially on the right side, and in the anterior lumbar region was felt an oval mass, quite dull on percussion, and offering an obscure sense of fluctuation on palpation. She had passed no urine for several hours. She continued to get worse for three days, not passing more than a teaspoonful of water daily. She was delirious, her tongue was yellowish, her pulse very small, and she was drowsy. On the third day she passed a pint of water. On the fourth day there was retention of urine, and three pints of water were drawn off by the catheter. The water passed on the first occasion was not seen, being mixed with menstrual fluid. The urine drawn off by the catheter was light-coloured, acid, containing a small quantity of albumen, and of specific gravity 1013. She soon recovered her usual health, and since that time has had occasional recurrences of pain and increased swelling in the lumbar region; during these attacks her urine has been scanty, but has been passed in unusually large quantities as the pain has subsided, 
She has not again had entire suppression of urine since 1855.

Mr. Joseph Thomson reports a case of hydronephrosis in an adult, who was several times tapped ('Path. Society's Transactions,' vol. xiii, page 128). 